Bond University

Research Repository

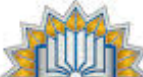 \\ *** BOND \\ UNIVERSITY
}

\section{Using Salivary Cortisol as an Objective Measure of Physiological Stress in People With Dementia and Chronic Pain: A Pilot Feasibility Study}

Pu, Lihui; Todorovic, Michael; Moyle, Wendy; Jones, Cindy

Published in:

Biological Research for Nursing

DOI:

$10.1177 / 1099800420934543$

Licence:

Other

Link to output in Bond University research repository.

Recommended citation(APA):

Pu, L., Todorovic, M., Moyle, W., \& Jones, C. (2020). Using Salivary Cortisol as an Objective Measure of Physiological Stress in People With Dementia and Chronic Pain: A Pilot Feasibility Study. Biological Research for Nursing, 22(4), 520-526. https://doi.org/10.1177/1099800420934543

\section{General rights}

Copyright and moral rights for the publications made accessible in the public portal are retained by the authors and/or other copyright owners and it is a condition of accessing publications that users recognise and abide by the legal requirements associated with these rights.

For more information, or if you believe that this document breaches copyright, please contact the Bond University research repository coordinator 


\title{
Using salivary cortisol as an objective measure of physiological stress in people with dementia and chronic pain: A pilot feasibility study
}

\begin{abstract}
Background: Pain experienced by people with dementia can elevate stress. Although salivary cortisol is used as a biomarker of stress in people with dementia, there are few studies reporting the feasibility of collection methods to assess salivary cortisol in nursing home residents with dementia and chronic pain. There is a need to extend research on physiological benefits using objective measures of physiological stress, such as salivary cortisol, in people with dementia and chronic pain.
\end{abstract}

Objective: To explore the feasibility of collecting cortisol via salivary swab as an indicator of stress in people with dementia and chronic pain.

Methods: Forty-three residents aged $\geq 65$ years and living with dementia and chronic pain were randomized to the PARO group (individual, non-facilitated, 30-minute sessions, five days per week for six weeks) or a usual care group using a computer-generated random number. The salivary cortisol was collected in the early morning before the intervention (week 0) and at the completion of the intervention (week 6) for comparison.

Results: There were multiple challenges associated with saliva collection and analysis within this study. These issues included cognitive impairment of participants; obtaining repeated samples with adequate saliva volume for assay; and the overall cost. Ultimately, adequate saliva was collected from only eight participants (both pre- and post-intervention) for assay and subject to quantitative analysis.

Conclusion: Considering the multiple challenges of obtaining valid saliva samples, salivary cortisol may not be a feasible biomarker of physiological stress in people with dementia and chronic pain.

Keywords: stress, cortisol, dementia, chronic pain, PARO, social robot 


\section{Introduction}

People with dementia frequently experience elevated stress due to unmet needs, such as pain or discomfort (Cohen-Mansfield, 2013; Sharp, 2019). Pain remains mostly unrecognized and untreated in people with dementia (Douglas, Haydon, \& Wollin, 2016) due to the reduced capacity to verbalize pain experience as well as the limited pain-related knowledge and lack of adequate skills possessed by the care-staff to observe and assess pain (Liu, 2014). Unrecognized and untreated pain may trigger a physiological stress response that includes increased secretion of cortisol (Hannibal \& Bishop, 2014), which is an important glucocorticoid hormone in humans that reflects Hypothalamus-Pituitary-Adrenal (HPA) axis adaptation to stressors (Hellhammer, Wust, \& Kudielka, 2009). Cortisol is predominantly $(90 \% \sim 95 \%)$ bound to proteins in the blood, and only unbound free cortisol $(5 \% \sim 10 \%)$ circulates as biologically active hormone (Kudielka, Hellhammer, \& Kirschbaum, 2000). Evidence shows that there is a high correlation between salivary cortisol levels and unbound free cortisol levels in plasma and serum (Gozansky, Lynn, Laudenslager, \& Kohrt, 2005; Reid, Intrieri, Susman, \& Beard, 1992). Therefore, the assessment of salivary cortisol levels to detect pain-related stress may be a convenient, repeated, and minimally invasive approach, especially for people with dementia living in residential aged care facilities (RACFs).

There is an increasing interest in using salivary cortisol as an objective measure of physiological stress in people with dementia living in RACFs (D'Cunha et al., 2019; Kwan, Leung, \& Lai, 2017; Venturelli et al., 2016). The use of different collection methods, sampling frequencies, and analytical approaches to assess salivary cortisol levels have been reported. Different collection practices tend to reflect the desired outcome measure, with some studies collecting saliva once per day (Kwan et al., 2017) while others multiple times per day (D'Cunha et al., 2019; Venturelli et al., 2016). Outcome measures included baseline cortisol levels assessed in the morning (Kwan et al., 2017), cortisol variation with circadian 
rhythm (Venturelli et al., 2016), or cortisol ratio (comparing waking to evening collections) (D'Cunha et al., 2019).

To date, there are limited studies which have reported on the desired measurable outcome and feasibility of appropriate collection methods to assess salivary cortisol in people with dementia and chronic pain living in RACFs. Woods et al. (2008) previously collected samples at multiple timepoints throughout the day to determine range and variability in nursing home residents with advanced dementia. They reported that it was feasible to assay $94 \% \sim 98 \%$ saliva samples collected. In contrast, Kwan et al. (2017) reported that $54.8 \%$ of samples were missing mainly due to an insufficient volume of saliva collection and hydration was the most important influencing factor for the missing data. Measuring stress hormones including cortisol, through saliva samples from dementia residents was challenging as only two (ten in total) residents had pre/post valid saliva samples for analysis (Bourne et al., 2019). Reasons for less saliva being collected included the method of saliva collection, time of the day, high temperature causing dehydration, procedures and personnel present (Bourne et al., 2019). The feasibility of using salivary cortisol as an objective measure of physiological stress in people with dementia and chronic pain remains to be determined.

Social robots, such as the robotic seal PARO, may have the potential to decrease corresponding cortisol levels (Jøranson, Pedersen, Rokstad, \& Ihlebæk, 2015) in people with dementia. However, Liang et al. (2017) found no significant differences in salivary cortisol levels between participants with dementia with a PARO intervention or usual care. To our knowledge, the feasibility of a PARO intervention on salivary cortisol has yet to be investigated in people with dementia who are experiencing chronic pain. Therefore, we set out to explore the feasibility of using salivary cortisol as an objective measure of physiological stress in people with dementia and chronic pain living in RACFs. Preliminary results from this study may help clinicians and researchers consider future collection and use 
of salivary cortisol of people with dementia and chronic pain and this could be informative for future studies to understand the physiological effects of PARO.

\section{Method}

This study was performed as part of a pilot randomized controlled trial, exploring the effect of a social robot (PARO) intervention for people with dementia living in RACFs in Australia. PARO, a therapeutic robotic seal, can be described as a social robot to enhance communication, socialisation, and emotional connection for people living with dementia. It has four senses of sight, hearing, balance and tactile to respond and communicates with users by moving or making a sound (Wada, Shibata, Saito, \& Tanie, 2003). Residents allocated to the intervention group received an individualized 30-minute PARO intervention from Monday to Friday for six weeks while those in the control group received usual care. Details of the PARO intervention and participant recruitment have been published elsewhere (blinded for review).

The feasibility of this study was guided by the Bowen Feasibility Framework (Bowen et al., 2009), including (1) acceptability (percentage of participants' adherence to the the protocol of salivary collection); (2) implementation (percentage of the collected samples for further assay); (3) practicality (cost); and (4) efficacy (effects of the intervention). The time, duration and attempts for saliva collection from each participant as well as reasons for failure were also recorded. This study was reviewed and approved by the University Human Research Ethics Committee (2017/774) and study sites. This study was prospectively registered with the Australia New Zealand Clinical Trials Registry (ACTRN12618000082202).Written informed consent was obtained from participants, if capable, or from their legally authorized representatives. Assent from participants was also sought before every intervention session.

\section{Data collection}


Socio-demographic information of participants was assessed at baseline. Cognitive status was assessed using the Mini-Mental State Examination (MMSE). Medical diagnosis and medications were audited from medical records.

Considering the feasibility nature of this study and to minimize subject burden, salivary samples were collected in the early morning of week 0 (before the intervention) and week 6 (after the intervention). First, the student researcher was trained and saliva collection was practiced with a registered nurse with expertise in dementia care, and a researcher familiar with the procedure. Then, the student researcher met with participants and care staff to introduce the procedure before data collection. In addition, a research flyer with the introduction of the salivary cortisol collection was provided to both the participant and care staff to help them become familiar with the procedure. Third, during the data collection, care staff were asked to accompany participants for assistance. If any participant did not want to open their mouth for the saliva collection three times, it was recorded as not being able to obtain a saliva sample from the participant.

Saliva samples were collected at the beginning of the study (pre-intervention at week 0) and the completion of the study (post-intervention at week 6). To control for confounding factors, such as eating, timing of collection, and exercise, all salivary cortisol was collected at a fixed time point within one hour of waking and before oral care, breakfast, and all forms of activities. Samples were extracted using the Salimetrics Children Swab collection kit (Salimetrics, PA. Item no. 5001.06). The Salimetrics oral swab is considered as the goldstandard device for saliva collection in the adult population as it is minimally invasive and easy to use (Tryphonopoulos, Letourneau, \& Azar, 2013). The swab is an elongated cylindrical synthetic swab $(8 \mathrm{~mm} \times 125 \mathrm{~mm})$ that can be held at one end by the researcher to avoid the risk of choking in people with dementia. The swab was placed in the participants' mouth under the tongue for at least two minutes (Salimetrics, 2015). All 
samples were collected within two hours (from 6:30 am to 8:30 am) to minimise potential diurnal/circadian influence. Collected saliva samples were kept in Salimetrics storage tubes and immediately stored in ice (below $4^{\circ} \mathrm{C}$ ) until they were transported to the laboratory and frozen at $-80^{\circ} \mathrm{C}$ until measurement readings were performed. All collected samples were deidentified and processed concurrently within a laboratory setting. Invalid samples with an extremely low salivary cortisol level and those that exceeded the calibrated range were discarded. This study used the most common method for measuring salivary cortisol - an enzyme immunoassay (EIA)/enzyme-linked immunoassay (ELISA) (Cortisol EIA Kit, Item no. 5001.02, Salimetrics). All measurements were performed in duplicates by an independent researcher who was blinded to the group allocation. The average coefficient of variation between duplicates equalled $1.11 \%$ (range $0.04 \%-3.6 \%$ ).

\section{Data analysis}

Descriptive statistics were computed to evaluate patient characteristics and the level of salivary cortisol (i.e., mean, standard deviation, frequency). Feasibility of participants' adherence and valid samples for assay was described as percentages. Repeated measures analysis of covariance (ANCOVA) was used to explore the effect of the PARO intervention on the level of saliva cortisol adjusting for baseline values. Statistical significance was set at $\mathrm{p}<0.05$. Analyses were carried out using IBM SPSS Statistics for Windows, Version 25.0. Armonk, NY: IBM Corp.

\section{Results}

Forty-three residents from three RACFs were randomly allocated to the intervention group $(n=21)$ and the control group $(n=22)$. The attrition rate of participants was $7.0 \%$ with three drop-outs due to death $(n=2)$ or loss of interest $(n=1)$. All participants were reported to experience chronic pain ( $\geq 3$ months) and had at least one pain-related condition. Among them, over half (55.8\%) residents had advanced dementia with an MMSE score lower than 
11. Antidepressants and antipsychotics were prescribed respectively to $20(46.5 \%)$ and 12 (27.9\%) participants (see Table 1). Further details of participants' characteristics are reported in a previously published paper (blinded for review).

\section{Saliva collection - Acceptability}

Table 2 shows the acceptability outcomes. A total of $43(100 \%)$ samples were collected before the intervention, and $38(88.4 \%)$ samples were collected at week six. The participant adherence rate was satisfactory. Most residents (95\%) agreed to put the swab in their mouth for two minutes with only two participants consistently refusing to undergo the saliva collection process at week six. An additional three samples were missing due to deceased participants $(n=2)$ and one resident was sent home on the day of data collection $(n=1)$.

\section{Samples for assay - Implementation}

Among the 81 available samples, $52(64.2 \%)$ samples were discarded due to inadequate saliva volume. Twenty-nine (35.8\%) samples (12 from pre-intervention and 17 from postintervention) from 21 participants had an adequate amount of saliva to perform the ELISA. However, only eight participants had both pre/post samples for assay and were subjected to quantitative analysis. The difference in the rate of valid saliva samples was not significant between the two groups, and it was not significantly related to age, gender, MMSE score and types of medication use.

\section{Cost - Practicality}

The cost of consumables to obtain 89 samples (Saliva Bio's Children's Swab, storage tube, labels, storage box, gloves, and the cortisol EIA kit) was around AUD 2000 in this study. The effect of PARO intervention on stress levels - Efficacy

Among the eight participants who had both pre and post saliva for assay, there were no significant differences between the two groups at baseline with respect to age, gender, MMSE score and medication use. The cortisol level at baseline for the intervention group 
$(\mathrm{n}=5)$ and control group $(\mathrm{n}=3)$ was $0.42(0.06) \mathrm{ug} / \mathrm{dL}$ and $0.44(0.07) \mathrm{ug} / \mathrm{dL}$. The cortisol level for two groups at week six was $0.44(0.02) \mathrm{ug} / \mathrm{dL}$ and $0.39(0.02) \mathrm{ug} / \mathrm{dL}$ (Table 2). The small sample size was inadequate for statistical analysis and conclusions cannot be drawn from these limited results.

\section{Discussion}

Several difficulties in collection and analysis were encountered when measuring cortisol in pre/post saliva samples in people with dementia living in RACFs. Our study had a much lower sample quality than previously reported studies (Kwan et al., 2017; Woods et al., 2008). This may be explained by the different degrees of cognitive impairment of participants as more people with severe dementia were included in this current study. Second, almost half of the participants $(46.5 \%)$ in this study had been prescribed antidepressants, which might reduce saliva production (Woods et al., 2008). Additionally, participants in Woods' (2008) study were allowed to chew on the cotton pledget for at least 1 minute which significantly improved the volume of collected saliva. A previous study reported that saliva secretion rate could be increased by chewing stimulation (van der Putten, Brand, De Visschere, Schols, \& de Baat, 2013). However, this may alter the accuracy of the results and could potentially dislodge food particulates that could also alter results (Salimetrics, 2015). Therefore, participants were not asked to chew on the cotton pledget in our study. We also encountered several methodological difficulties with saliva collection in people with dementia: (1) acceptability - cognitive impairment of participants; (2) implementation - obtaining pre/post samples with adequate saliva volume for assay; (3) practicality - cost; and (4) efficacy - the effect of PARO intervention on the level of salivary cortisol.

\section{(1) Acc ability - cognitive impairment of participants}

People with advanced dementia who may not fully understand the nature of this technique and the requirement that no food be consumed before collection may contaminate the 
collected sample. Poor oral hygiene (e.g., food debris, bleeding gums) for people with dementia living in RACFs is another factor for potential contamination (Delwel et al., 2018). Furthermore, although the researchers explained the sample collection approach to familiarize participants with the procedure and encourage them to open their mouth, participants with advanced dementia who expressed anxiety and fear about the safety and nature of this procedure may experience additional stress, and this could potentially modify the level of stress biomarker to be evaluated. This highlights that collaboration with care staff is essential to ensure participants are informed of the data collection procedure. Future studies may opt for training of care staff to engage in the collection of the saliva to help reassure, comfort, and guide residents during the procedure.

Despite previous studies recommending the use of the oral swab collection method, Bourne et al. (2019) reported challenges with using oral swabs to collect saliva in people with dementia and recommended the use of the passive drool method (McKune, Bach, Semple, \& Dyer, 2014) to gather sufficient saliva. This method has the advantage of a visual assessment of the collected volume of saliva; however, it could be challenging in people with advanced dementia who may find it testing to understand and follow instructions, and to produce adequate amounts of saliva. There is currently a small selection of portable or point-of-care diagnostic tools (Malon, Sadir, Balakrishnan, \& Córcoles, 2014; Yamaguchi, Katagata, Tezuka, Niwa, \& Shetty, 2014), which allow noninvasive, quantitative, and automated analysis of human salivary cortisol levels that facilitate timely diagnosis. Alternatively, wearable technologies could also monitor cortisol level from low volume (1-5 uL) of human sweat using portable biosensors (Kinnamon, Ghanta, Lin, Muthukumar, \& Prasad, 2017). Researchers may opt to test the feasibility of these in future studies.

\section{(2) Implementation - obtaining pre/post samples with adequate saliva volume for assay}

Evidence demonstrates that cortisol level varies throughout the day, following a diurnal 
rhythm, with serum levels highest in the early morning (30-45 minutes after wake time) and decreasing throughout the day (Hulett, Fessele, Clayton, \& Eaton, 2019). It is recommended to collect samples with multiple measurement points throughout a 24-hour time period, over at least two days, to obtain accurate baseline and subsequent readings (Woods \& Mentes, 2011). However, evidence suggests that the variations in basal cortisol levels between two consecutive days are small (Kwan, Leung, \& Lai, 2016). In this current study, the salivary cortisol was collected in the early morning within two-hours on a single day. However, the timing of data collection at week 0 and week 6 was not exactly the same as it was dependent on the participants time of waking.

Saliva samples were collected using the SalivaBio Children's Swab, a synthetic swab specifically designed to improve volume collection and increase participant compliance by eliminating any choking hazards. It is validated for use with saliva collection in people with dementia (Williams et al., 2016). While the swab was placed in the residents' mouth under the tongue for at least two minutes - a time period longer than the 60-90 seconds instructed by the kit - the majority $(64.2 \%)$ of the samples were discarded due to inadequate saliva volume for further assay. This was the most significant and rate-limiting issue faced from the outset of this study. Several factors may contribute to this issue. First, reduced salivary secretion (hyposalivation) and sensations of oral dryness (xerostomia) are associated with older age (Desoutter et al., 2012) and the decrease of saliva production in the salivary glands (Ortega-Martínez, Cedeño-Salazar, Requena, Tost, \& Lluch, 2014). Second, the use of medications, such as antipsychotics, antidepressants, and anti-inflammatory medications, which interfere with the autonomic nervous systems, play an important role in the reduction of saliva flow (Leal et al., 2010). These medications are commonly prescribed to people with dementia, and this may further increase the risk of hyposalivation and xerostomia (Tan, Lexomboon, Sandborgh-Englund, Haasum, \& Johnell, 2018). Evidence also shows that 
people with Alzheimer's disease produce significantly less saliva per minute than those without the disease (Aragón et al., 2018). Additionally, psychological factors, such as anxiety and depression, which are common for residents living in RACFs, could contribute to xerostomia (Gholami, Hosseini Sabzvari, Razzaghi, \& Salah, 2017). These factors can account for large amounts of missing data and limit the validity of the results.

\section{(3) Prac ality-cost}

The cost of collecting and analyzing salivary cortisol in people with dementia needs to be considered. We only calculated the cost of consumables to obtain salivary samples in this study, and the cost of saliva collection, cortisol assay, and data analysis would also lead to additional costs. Overall, to obtain adequate samples, collected over multiple time points and days, both pre and post-intervention would significantly increase the relative cost for this measure.

\section{(4) Effi - the effect of PARO intervention on the level of salivary cortisol}

Due to the low valid saliva sample collection rate, interpretation of the salivary cortisol result was a challenge. The small sample size inhibited the statistical power to detect any significant differences between groups. Therefore, conclusions on the effect of PARO to reduce salivary cortisol levels in people with dementia needs further exploration. The validity of the results could be improved with a larger sample size and better saliva collection methods. Limitations The use of a single daily measure of salivary cortisol may be inadequate to capture the cortisol diurnal cycle and salivary collection within the first hour of waking did not avoid the

confounding influence of the cortisol awakening response. Future studies should standardize the collection time based upon when the participant awakens. To fully capture or reflect the diurnal rhythms of cortisol release, ideally multiple samples should be collected per day for each participant. Participants with corticosteroid medications were also not excluded as it is 
expected that the majority of residents living in RACFs receive pharmacological treatment. Additionally, the varied time of data collection before and after the intervention may have an extraneous influence on the results. Despite these limitations, the reported findings remain valuable considering the nature of this feasibility study and the difficulty in collecting objective data from people with dementia and chronic pain living in RACFs.

\section{Implications for future research}

Although salivary cortisol analysis offers some advantages such as ease of data collection and a relatively non-invasive approach in comparison to the collection of blood or cerebral spinal fluid, it should be noted that there is currently a lack of standard salivary collection procedures for people with dementia. Issues such as the timing, frequency, collection device, and potential confounding factors such as age-related hyposalivation and xerostomia, medication use and medical conditions, can significantly impact the rigor of this measure. More research is required in this area to determine a feasible and valid biomarker to measure physiological stress in people with dementia and chronic pain. For example, a new wristband called DemaWare@NH (Kikhia et al., 2016), which measures Galvanic Skin Response (GSR), accelerometers (for capturing motion), skin temperature, environment temperature and environment light, are feasible to measure stress level in people with dementia. Besides, biomarker-based evaluation of social robot interventions for people with dementia is still a relatively new and evolving concept which can serve to complement qualitative and mixedmethods research.

\section{Conclusion}

Considering the challenges of obtaining valid saliva samples, salivary cortisol may not be a suitable biomarker or an objective measure of physiological stress in people with dementia and chronic pain. The preliminary results based on eight participants could not quantify the physiological effects of PARO on stress and further research is needed to further the 
understanding.

\section{Conflicts of interest}

All authors declare no financial, personal, or potential conflicts of interest.

\section{Acknowledgements}

The researchers are grateful to the participants and nursing home healthcare staff who assisted with participant selection and data collection.

\section{References}

Aragón, F., Zea-Sevilla, M. A., Montero, J., Sancho, P., Corral, R., Tejedor, C., . . . Albaladejo, A. (2018). Oral health in Alzheimer's disease: a multicenter case-control study. Clinical Oral Investigations, 22(9), 3061-3070. doi:10.1007/s00784-018-2396-z

Bourne, P., Camic, P., Crutch, S., Hulbert, S., Firth, N., \& Harding, E. (2019). Using Psychological and Physiological Measures in Arts-Based Activities in a Community Sample of People with a Dementia and Their Caregivers: A Feasibility and Pilot Study. Journal of Aging Studies and Therapies. doi:10.16966/jast.102

Bowen, D. J., Kreuter, M., Spring, B., Cofta-Woerpel, L., Linnan, L., Weiner, D., . . . Fernandez, M. (2009). How We Design Feasibility Studies. American journal of preventive medicine, 36(5), 452-457. doi:10.1016/j.amepre.2009.02.002

Cohen-Mansfield, J. (2013). Nonpharmacologic treatment of behavioral disorders in dementia. Current Treatment Options in Neurology, 15(6), 765-785.

D'Cunha, N. M., McKune, A. J., Isbel, S., Kellett, J., Georgousopoulou, E. N., \& Naumovski, N. (2019). Psychophysiological Responses in People Living with Dementia after an Art Gallery Intervention: An Exploratory Study. J Alzheimers Dis. doi:10.3233/jad-190784 Delwel, S., Binnekade, T. T., Perez, R. S. G. M., Hertogh, C. M. P. M., Scherder, E. J. A., \& Lobbezoo, F. (2018). Oral hygiene and oral health in older people with dementia: a comprehensive review with focus on oral soft tissues. Clinical Oral Investigations, 22(1), 93-108. doi:10.1007/s00784-017-2264-2

Desoutter, A., Soudain-Pineau, M., Munsch, F., Mauprivez, C., Dufour, T., \& Coeuriot, J. L. (2012). Xerostomia and medication: a cross-sectional study in long-term geriatric wards. $J$ Nutr Health Aging, 16(6), 575-579. 
Douglas, C., Haydon, D., \& Wollin, J. (2016). Supporting Staff to Identify Residents in Pain: A Controlled Pretest-Posttest Study in Residential Aged Care. Pain Management Nursing, 17(1), 25-37. doi:10.1016/j.pmn.2015.08.001

Gholami, N., Hosseini Sabzvari, B., Razzaghi, A., \& Salah, S. (2017). Effect of stress, anxiety and depression on unstimulated salivary flow rate and xerostomia. Journal of dental research, dental clinics, dental prospects, 11(4), 247-252.

doi:10.15171/joddd.2017.043

Gozansky, W., Lynn, J., Laudenslager, M., \& Kohrt, W. (2005). Salivary cortisol determined by enzyme immunoassay is preferable to serum total cortisol for assessment of dynamic hypothalamic-pituitary-adrenal axis activity. Clinical endocrinology, 63(3), 336-341.

Hannibal, K. E., \& Bishop, M. D. (2014). Chronic stress, cortisol dysfunction, and pain: a psychoneuroendocrine rationale for stress management in pain rehabilitation. Physical Therapy, 94(12), 1816-1825. doi:10.2522/ptj.20130597

Hellhammer, D. H., Wust, S., \& Kudielka, B. M. (2009). Salivary cortisol as a biomarker in stress research. Psychoneuroendocrinology, 34(2), 163-171.

doi:10.1016/j.psyneuen.2008.10.026

Hulett, J. M., Fessele, K. L., Clayton, M. F., \& Eaton, L. H. (2019). Rigor and Reproducibility: A Systematic Review of Salivary Cortisol Sampling and Reporting Parameters Used in Cancer Survivorship Research. Biological Research for Nursing, 21(3), 318-334. doi:10.1177/1099800419835321

Jøranson, N., Pedersen, I., Rokstad, A. M. M., \& Ihlebæk, C. (2015). Effects on symptoms of agitation and depression in persons with dementia participating in robot-assisted activity: A cluster-randomized controlled trial. Journal of the American Medical Directors Association, 16(10), 867-873. doi:10.1016/j.jamda.2015.05.002.

Kikhia, B., Stavropoulos, T. G., Andreadis, S., Karvonen, N., Kompatsiaris, I., Sävenstedt, S., .. . Melander, C. (2016). Utilizing a Wristband Sensor to Measure the Stress Level for People with Dementia. Sensors (Basel, Switzerland), 16(12), 1989. doi:10.3390/s16121989

Kinnamon, D., Ghanta, R., Lin, K.-C., Muthukumar, S., \& Prasad, S. J. S. r. (2017). Portable biosensor for monitoring cortisol in low-volume perspired human sweat. 7(1), 1-13.

Kudielka, B. M., Hellhammer, D., \& Kirschbaum, C. (2000). Sex differences in human stress response. In Stress Consequences: Mental, Neuropsychological and Socioeconomic (Vol. 3): Academic Press San Diego. 
Kwan, R. Y., Leung, M. C., \& Lai, C. K. (2016). The Effect of Acupressure on Agitation and Salivary Cortisol in People with Dementia: A Pilot Study. Journal of Alternative and Complementary Medicine, 22(11), 903-910. doi:10.1089/acm.2016.0062

Kwan, R. Y. C., Leung, M. C. P., \& Lai, C. K. Y. (2017). A Randomized Controlled Trial Examining the Effect of Acupressure on Agitation and Salivary Cortisol in Nursing Home Residents with Dementia. Dement Geriatr Cogn Disord, 44(1-2), 92-104. doi:10.1159/000478739

Leal, S. C., Bittar, J., Portugal, A., Falcão, D. P., Faber, J., \& Zanotta, P. (2010). Medication in elderly people: its influence on salivary pattern, signs and symptoms of dry mouth. Gerodontology, 27(2), 129-133. doi:10.1111/j.1741-2358.2009.00293.x

Liang, A., Piroth, I., Robinson, H., MacDonald, B., Fisher, M., Nater, U. M., . . Broadbent, E. (2017). A pilot randomized trial of a companion robot for people with dementia living in the community. Journal of the American Medical Directors Association, 18(10), 871878. doi:10.1016/j.jamda.2017.05.019

Liu, J. Y. W. (2014). Exploring nursing assistants' roles in the process of pain management for cognitively impaired nursing home residents: a qualitative study. Journal of Advanced Nursing, 70(5), 1065-1077. doi:10.1111/jan.12259

Malon, R. S. P., Sadir, S., Balakrishnan, M., \& Córcoles, E. P. (2014). Saliva-based biosensors: noninvasive monitoring tool for clinical diagnostics. BioMed Research International, 2014, 962903-962903. doi:10.1155/2014/962903

McKune, A. J., Bach, C. W., Semple, S. J., \& Dyer, B. J. (2014). Salivary cortisol and alphaamylase responses to repeated bouts of downhill running. Am J Hum Biol, 26(6), 850-855. doi:10.1002/ajhb.22605

Ortega-Martínez, J., Cedeño-Salazar, R., Requena, C., Tost, M., \& Lluch, A. (2014). Alzheimer's disease: oral manifestations, treatment and preventive measures. Journal of Oral Research(3), 184-189\%V 183. doi:10.17126/joralres.2014.043

Reid, J. D., Intrieri, R. C., Susman, E. J., \& Beard, J. L. (1992). The relationship of serum and salivary cortisol in a sample of healthy elderly. Journal of Gerontology, 47(3), P176-P179.

Salimetrics. (2015). Saliva Collection and Handling Advice, 3rd Edition. Retrieved from https://salimetrics.com/saliva-collection-handbook/

Sharp, B. K. (2019). Stress as Experienced by People with Dementia: An Interpretative Phenomenological Analysis. Dementia (London), 18(4), 1427-1445. doi:10.1177/1471301217713877 
Tan, E. C. K., Lexomboon, D., Sandborgh-Englund, G., Haasum, Y., \& Johnell, K. (2018). Medications That Cause Dry Mouth As an Adverse Effect in Older People: A Systematic Review and Metaanalysis. Journal of the American Geriatrics Society, 66(1), 76-84. doi:10.1111/jgs.15151

Tryphonopoulos, P. D., Letourneau, N., \& Azar, R. (2013). Approaches to Salivary Cortisol Collection and Analysis in Infants. Biological Research for Nursing, 16(4), 398-408. doi:10.1177/1099800413507128

van der Putten, G. J., Brand, H. S., De Visschere, L. M., Schols, J. M., \& de Baat, C. (2013). Saliva secretion rate and acidity in a group of physically disabled older care home residents. Odontology, 101(1), 108-115. doi:10.1007/s10266-011-0054-X

Venturelli, M., Sollima, A., Ce, E., Limonta, E., Bisconti, A. V., Brasioli, A., . . Esposito, F. (2016). Effectiveness of Exercise- and Cognitive-Based Treatments on Salivary Cortisol Levels and Sundowning Syndrome Symptoms in Patients with Alzheimer's Disease. $J$ Alzheimers Dis, 53(4), 1631-1640. doi:10.3233/jad-160392

Wada, K., Shibata, T., Saito, T., \& Tanie, K. (2003, 20-24 July 2003). Psychological, physiological and social effects to elderly people by robot assisted activity at a health service facility for the aged. Paper presented at the Advanced Intelligent Mechatronics, 2003. AIM 2003. Proceedings. 2003 IEEE/ASME International Conference on.

Williams, C., Tappen, R., Wiese, L., Newman, D., Corbett, M., Pinos, S., . . Murray, B. (2016). Stress in Persons with Dementia: Benefits of a Memory Center Day Program. Archives of Psychiatric Nursing, 30(5), 531-538.

doi:https://doi.org/10.1016/j.apnu.2016.04.011

Woods, D. L., Kovach, C. R., Raff, H., Joosse, L., Basmadjian, A., \& Hegadoren, K. M. (2008). Using saliva to measure endogenous cortisol in nursing home residents with advanced dementia. Research in Nursing \& Health, 31(3), 283-294.

doi:10.1002/nur.20254

Woods, D. L., \& Mentes, J. C. (2011). Spit: saliva in nursing research, uses and methodological considerations in older adults. Biological Research for Nursing, 13(3), 320-327. doi:10.1177/1099800411404211

Yamaguchi, M., Katagata, H., Tezuka, Y., Niwa, D., \& Shetty, V. (2014). Automatedimmunosensor with centrifugal fluid valves for salivary cortisol measurement. Sensing and Bio-Sensing Research, 1, 15-20. doi:https://doi.org/10.1016/j.sbsr.2014.07.001 
Table 1 Participants information $(n=43)$

\begin{tabular}{lccc}
\hline Variables & $\begin{array}{c}\text { Total } \\
(\mathbf{n = 4 3 )}\end{array}$ & $\begin{array}{c}\text { Control group } \\
(\mathbf{n = 2 2})\end{array}$ & $\begin{array}{c}\text { Intervention group } \\
(\mathbf{n = 2 1})\end{array}$ \\
\hline Age $^{*}$ & $86.00 \pm 7.35$ & $85.55 \pm 5.80$ & $86.48 \pm 8.81$ \\
Gender & & & \\
Female & $30(69.8 \%)$ & $12(54.5 \%)$ & $18(85.7 \%)$ \\
Male & $13(30.2 \%)$ & $10(45.4 \%)$ & $3(14.3 \%)$ \\
Dementia subtypes & & & \\
Alzheimer's disease & $17(39.5 \%)$ & $8(36.4 \%)$ & $9(42.9 \%)$ \\
Vascular dementia & $5(11.6 \%)$ & $3(13.6 \%)$ & $2(9.5 \%)$ \\
Frontal-temporal dementia & $1(2.3 \%)$ & $1(4.5 \%)$ & $0(0.0 \%)$ \\
Dementia unspecified & $20(46.5 \%)$ & $10(45.5 \%)$ & $10(47.6 \%)$ \\
MMSE $\leq \mathbf{1 0}$ & $24(55.8 \%)$ & $9(40.9 \%)$ & $15(71.4 \%)$ \\
Verbal communication, yes & $32(74.4 \%)$ & $18(81.8 \%)$ & $14(66.7 \%)$ \\
Medication use, yes & & & \\
Antidepressants & & $9(40.0 \%)$ & $11(52.4 \%)$ \\
Antipsychotics & $20(46.5 \%)$ & $6(27.9 \%)$ & $6(27.3 \%)$ \\
Benzodiazepines & $12(27.9 \%)$ & $4(18.2 \%)$ & $2(9.1 \%)$ \\
Anticonvulsant & $6(16.3 \%)$ & $1(4.5 \%)$ & $3(14.3 \%)$ \\
\hline
\end{tabular}

Table 2 Feasibility outcomes

\begin{tabular}{|c|c|c|c|c|}
\hline \multirow{2}{*}{ Feasibility } & \multirow{2}{*}{ Outcomes of inter } & \multirow{2}{*}{ Groups } & \multicolumn{2}{|c|}{ Results } \\
\hline & & & Pre & Post \\
\hline Acceptability & $\begin{array}{l}\text { Adherence to } \\
\text { data collection }\end{array}$ & $\begin{array}{l}\text { Intervention } \\
(\mathrm{n}=21) \\
\text { Control }(\mathrm{n}=22)\end{array}$ & $\begin{array}{l}21(100 \%) \\
22(100 \%)\end{array}$ & $\begin{array}{l}20(95.2 \%) \\
18(81.8 \%)\end{array}$ \\
\hline Implementation & $\begin{array}{l}\text { Collect repeated } \\
\text { samples for assay }\end{array}$ & $\begin{array}{l}\text { Intervention } \\
(n=21) \\
\text { Control }(n=22)\end{array}$ & $\begin{array}{l}8(38.1 \%) \\
4(18.2 \%)\end{array}$ & $\begin{array}{l}8(40.0 \%) \\
9(47.4 \%)\end{array}$ \\
\hline Practicality & Cost & AUD 2000 & & \\
\hline \multirow{3}{*}{ Efficacy } & & & Mean(SD) & Mean(SD) \\
\hline & $\begin{array}{l}\text { Effects of } \\
\text { intervention }\end{array}$ & $\begin{array}{l}\text { Intervention } \\
(n=5)^{*}\end{array}$ & $0.42(0.06)$ & $0.44(0.02)$ \\
\hline & & Control $(\mathrm{n}=3)^{*}$ & $0.44(0.07)$ & $0.39(0.02)$ \\
\hline
\end{tabular}

